\title{
HiSTORIA NACIONAL E INTERCULTURALIDAD: APUNTES CHIAPANECOS
}

\author{
National History and Interculturality: notes from Chiapas
}

Elisa Cárdenas-Ayala

Resumen: El artículo da cuenta de la reflexión que acompañó la realización de un taller de escritura de historias con jóvenes chiapanecos, en torno a algunos de los retos que enfrenta la escritura de la historia nacional para renovarse y hasta dónde puede dialogar con el concepto de interculturalidad en busca de vías fructíferas de renovación. El texto expone elementos de la experiencia misma del taller y algunos de sus resultados más relevantes en términos de dicha reflexión.

Palabras clave: escritura de la historia, nación, interculturalidad.

Abstract: This article gives an account of the reflection that accompanied a writing workshop among young students in Chiapas. It surrounds some of the challenges facing the national history writing and how far can go the dialogue within national history and the intercultural perspective. The text exposes elements of the workshop experience itself and some of its most important results.

Keywords: history writing, nation, interculturality.

Elisa Cárdenas Ayala, doctora en Historia por la Universidad Paris 1 Panthéon-Sorbonne. Profesora-investigadora del Departamento de Estudios sobre Movimientos Sociales de la Universidad de Guadalajara. Temas de especialización: revolución mexicana, procesos de secularización y construcción nacional en América Hispánica, escritura de la historia nacional mexicana. Correo electrónico: cardenasayala@gmail.com.
Enviado a dictamen: 16 de noviembre de 2012

Aprobación: 16 de enero de 2013

Revisiones: 1 


\section{Introducción}

Estas páginas buscan dar cuenta de la reflexión que acompañó la realización de un taller de escritura de historias con jóvenes chiapanecos, reflexión que discurrió en torno a algunos de los retos que enfrenta la escritura de la historia nacional para vivificarse y hasta dónde ésta puede dialogar con el concepto de interculturalidad en busca de vías fructíferas de renovación. El texto expone elementos de la experiencia misma del taller y algunos de sus resultados más relevantes en términos de dicha reflexión.

Así, el artículo explora el cruce de dos realidades cuya compatibilidad no es evidente: la nación como construcción política, con las representaciones frecuentemente uniformadoras que la acompañan, y el carácter intercultural de la nación. Ambas realidades se encuentran, cuando no entrechocan, en ese terreno en transformación constante que es la práctica de la escritura de la historia.

\section{Historia y nación: realidades discursivas, realidades desencantadas}

Andando ya el siglo XXI, a nadie sorprende que los términos "historia" y "nación" traduzcan, como hace ya varias décadas se ha venido insistiendo, realidades fundamentalmente discursivas - no por ello, empero, menos reales-. Simultáneamente, cualquiera puede constatar que, en ambos casos, estamos ante un par de sonoros y sonados desencantos, si bien no necesariamente de fracasos.

$\mathrm{Y}$ es que ambos discursos tuvieron su momento de encanto y apogeo en la segunda mitad del siglo XIX occidental, con esa Historia empeñada en trazar pasados y destinos nacionales, un modelo que cundió a ambos lados del Atlántico y dio incontables frutos historiográficos y políticos.

Que los conceptos de historia y nación fueron meteoros lanzados con fuerza mayúscula o, al menos, con intención mayúscula, lo muestra el hecho de que la trayectoria del desencantamiento de ambos tuvo aliento para recorrer todo el siglo XX y aún sirvió para dar pie a una de las propuestas de caracterización cronológica de dicho siglo que mayor eco ha tenido (Hobsbawm, 1994).

En la actualidad, sin embargo, costaría mantener su escritura con mayúscula: ni la historia como disciplina ni la historia como acontecer la resisten ya, y la historia como escritura tal vez nunca haya tenido la pretensión de ostentarla, Por su parte, la nación se muestra hace tiempo despojada del ropaje de solemnidad con que precisamente el discurso de la historia — si acaso empujada por la oratoria política de ocasión - alguna vez la revistiera.

Ambas trayectorias tienen momentos de paralelismo importantes, si bien en sentido inverso: el mundo desencantado de la nación es el de la historia secularizada (Koselleck, 2003) - la forma más literal de desencantamiento-, un proyectil lanzado con enorme potencia en esa misma segunda mitad del siglo XIX, en sentido historiográfico el momento más tangible de la nación endiosada. ${ }^{2}$ No pocas paradojas acompañan a este paralelismo, siendo de destacar aquella que hace que con los instrumentos de una disciplina científica naciente se contribuya a tejer la nación como mito. Las historias nacionales se cuentan, como se sabe, entre los primeros productos de una historiografía con pretensiones científicas.

Por lo demás, con respecto a esa larga trayectoria de desencanto, las ciencias sociales no nos han dicho aún - si cupiera pensar que algún día nos lo diránen qué momento empezó a diluirse la nación como sentimiento. Desde la disciplina de la historia pueden hacerse algunas hipótesis, ${ }^{3}$ aunque difícilmente suplirían éstas a una encuesta sociológica amplia sobre la vigencia del sentimiento nacional en generaciones escalonadas, cuya oportunidad seguramente está perdiéndose. Una encuesta para la cual el discurso educativo y sus modulaciones y repercusiones tendrían 
valor fundamental y que permitiría evaluar la vigencia de la nación como realidad simbólica de alcance colectivo.

Nacida en las últimas décadas del siglo XX, y de reciente práctica en elámbitoacadémicoiberoamericano, la semántica histórica — conocida también como historia de los conceptos - ha contribuido a mostrar de qué manera el desencanto de la nación y de la historia no es producto de un mero desgaste del discurso, sino, y sobre todo, de cambios históricos que inciden en y demuestran la historicidad irreductible de ambos conceptos (Koselleck, 2004; Fernández Sebastián, 2009: 549-691 y 849-977).

\section{Límites, retos y esperanzas}

Desde el último tercio del siglo XX y hasta nuestros días, la historia como disciplina se ha visto atravesada por debates que abordan frontalmente su calidad discursiva irreductible y que cuestionan las condiciones de producción y reproducción de este discurso de la mano de los avances de la lingüística, la psicología y la reflexión filosófica. ${ }^{4}$ No cabe entonces duda de que la producción de saber histórico es un proceso de escritura en el que interviene ampliamente la subjetividad de quien escribe, si bien está apoyado en conocimientos técnicos y socialmente condicionados (Certeau, 1978; Ricoeur, 2000: 165-369).

Cierto que la discusión en torno al carácter y condiciones de elaboración del discurso historiográfico cuestiona el estatuto de verdad del saber histórico al resaltar el papel del entorno, del individuo y de los valores propios en la producción de un saber cuya vía de expresión por excelencia es el lenguaje escrito. Esto, que muchas veces fue interpretado con precipitación como un cuestionamiento que alejaba a la disciplina del envidiable estatuto de "ciencia", en el fondo no hace sino acercarla al conjunto vasto de las ciencias que también han visto relativizada la validez del conocimiento que producen y con ello han reafirmado su carácter de saberes socialmente producidos y sobre los cuales existe una ineludible obligación de reflexionar (Bourdieu, 2001).

Sin que se considere ya que la disciplina está en crisis, como sugería el debate en los años noventa, el cambio de siglo se acompañó de una discusión intensa, transatlántica, que ha ido acentuando la centralidad del sujeto, centralidad que se encuentra en tensión constante — como ya lo estaba en Ricoeur (2000) con el análisis crítico del testimonio y del lugar que en nuestras sociedades contemporáneas ocupa la memoria (Sarlo, 2005; Tischler Visquerra, 2005). Centralidad del sujeto que también subraya la del historiador y agudiza la reflexión sobre los límites de la producción de saber. Es la preocupación de Ginzburg por la retórica y la prueba (2000), y la de Hartog por la evidencia (2005).

La insistencia en el papel del sujeto como productor de saber social - en el sentido no sólo de un saber socialmente producido, sino también de un saber del que puede apropiarse la sociedad en su conjuntono debería minar la confianza en la capacidad de una disciplina para producir conocimiento válido sobre la realidad sino, antes bien, esperanzar en cuanto a las posibilidades de que una sociedad se apropie de saberes que son realidades simultáneamente individuales y colectivas, como en el caso que nos ocupa, la escritura de su historia. ${ }^{6}$

Más allá, cabe igualmente preguntarse hasta dónde es posible asumir en el campo de la historia, como en el de otras ciencias sociales, el reto lanzado por Pierre Bourdieu a los sociólogos desde su cátedra inaugural en el Collège de France en abril de 1982:

[...] la sociología de la sociología, que permite movilizar en contra de la ciencia que se está estableciendo los conocimientos de la ciencia ya establecida, es un instrumento imprescindible del método sociológico: se hace ciencia - y sobre todo sociología - tanto contra su formación como con ella (Bourdieu, 2002: 10). 
Una observación que, por cierto, el mismo Bourdieu llevaba en esa ocasión hasta el campo específico de la historia:

\section{Y sólo la historia puede librarnos de la historia. Así es como la historia social de la ciencia social, siempre y cuando se conciba a sí misma también como una ciencia de lo inconsciente, en la gran tradición de epistemología histórica ilustrada por Georges Canguilhem y por Michel Foucault, es uno de los instrumentos más poderosos para desgajarse de la historia, es decir del influjo de un pasado incorporado que sobrevive en el presente o de un presente que, como el de las modas intelectuales, ya es pasado en el momento de su aparición (Bourdieu, 2002: 10).}

Sin embargo, más allá de sus condiciones de elaboración y de las posibilidades que la disciplina tiene para rebasar sus propias fronteras, importa subrayar que la historia, además de una escritura, es un saber del que sujetos concretos pueden apropiarse y, en esa misma medida, puede ser una escritura de la que sujetos concretos se apropien.

En tiempos en los que en nuestras sociedades se tiende a despreciar la relación con el pasado como una manera de desentenderse de los conflictos heredados, seguramente es preciso avanzar hacia nuevas formas de relación con la historia. Reflexionando sobre el siglo XX, lo escribía Hobsbawm en los términos siguientes:

The destruction of the past, or rather of the social mechanisms that link one's contemporary experience to that of earlier generations, is one of the most characteristic and eerie phenomena of the late twentieth century. Most young men and women at the century's end grow up in a sort of permanent present lacking any organic relation to the public past of the times they live in. This makes historians, whose business it is to remember what others forget, more essential at the end of the second millennium than ever before. But for that very reason they must be more than simply chroniclers, remembrancers and compilers, though this is also the historian's necessary function (Hobsbawm, 1994: 3).

\section{La historia nacional mexicana. En torno a su escritura}

Tras casi dos siglos de discurso "nacional", por paradójico que pueda parecer, aun desde la duda de que la nación exista, y por esa misma duda, la necesidad de renovar la escritura de su historia es patente. Desde el horizonte de la producción historiográfica —es decir, en cierta forma desde dentro de la disciplina- esta tarea es abordada de diversas maneras, con variadas estrategias y medios. Hace tiempo que nos alejamos de la historia oficial aunque la historiografía con intenciones pedagógicas a veces tenga aires de botín en disputa. Numerosos son los esfuerzos individuales que pugnan por ensanchar el caudal de la historia admitida como "nacional" y que obligan a preguntarse si, en el caso de que lo nacional sea un valor, entonces por quién o quiénes es atribuido y en ocasión de qué o en función de cuáles méritos. Además, no son pocas las empresas colectivas que intentan dotarnos de instrumentos generales de interpretación para un mejor conocimiento del conjunto y que también abordan lo nacional como objeto historiográfico.?

Desde otra perspectiva, y si se pretende ir más allá de la etiqueta de moda, el planteamiento actualmente en construcción de formas de educación intercultural - y en sentido amplio de políticas interculturales - ${ }^{8}$ obliga a interrogarse sobre las dinámicas de producción y de apropiación del discurso histórico en torno a la nación mexicana, y aún exige un replanteamiento de las mismas.

Sin duda es deseable la renovación de la escritura de la historia de la nación mexicana en un ejercicio que parta de su condición intercultural, no en la forma de un catálogo de culturas, que hace de éstas compartimentos 
estancos, sino atendiendo a los lugares y formas de interacción entre diferentes. ${ }^{9}$

Un primer esfuerzo tendría que ir en el sentido de un diálogo abierto con sujetos vivos que permita encontrar las prioridades temáticas, de enfoque e interpretativas, de unos, de otros y otros más.

Una escritura de la historia nacional que tenga por eje la condición intercultural de la nación estará menos lejos —es difícil decir más cerca- de lograr representaciones del pasado común en donde se reconozcan con dignidad quiénes son parte hoy hegemónica o subalterna, evidente o ignorada - de esta comunidad política, incluso en condición de excluidos. Aunque muchos esfuerzos han sido abonados desde la academia, estamos lejos de que la historia escrita sea un fenómeno de apropiación amplia.

Desde la perspectiva de los espacios "indios" sobre los que tiende a calcarse la interculturalidad, la formación de historiadores profesionales que se reconocen una identidad "india" apenas es reconocida, lo que no quiere decir que no existan depositarios privilegiados y reconstructores de historias de los pueblos bajo etiquetas que nuestro ejercicio profesional, de matriz occidental, ignora. Pero la acumulación de textos "indios" tampoco camina en dirección de una escritura intercultural, aunque sea, sin lugar a dudas, indispensable.

Si bien todo el país es terreno de relaciones interculturales de muy diversos tipos, es claro que la sustitución de una política indigenista por una intercultural tiende a confinar lo intercultural dentro de lo indio, lo que no sólo restringe las posibilidades de su desarrollo, sino que confiere al término "intercultural" una carga política paradójica en la medida en que lo hace portador de un impulso liberador - y aun de una referencia utópica-, al tiempo que lo calca sobre los espacios de lo "indio", probablemente reproduciendo el contenido colonial de esta categoría.

Los diálogos múltiples que son condición de una sociedad menos injusta no pueden dejar de lado la historia común. Y el diálogo sobre el pasado común, que naciones sobrevivientes de traumas colectivos mayores han reconocido como fundamental - pienso especialmente en las comisiones ad hoc de varias naciones sudamericanas-, no puede ser sólo materia de expertos, sobre todo porque en el universo de los expertos - que encima tiende a monoculturizarseestá sobrerrepresentado el punto de vista hegemónico.

Así, una escritura de la historia nacional que tenga como punto de partida la condición intercultural de la nación es indispensable, pues la historia de la nación no es única, sino múltiple, y en su multiplicidad contiene innumerables formas de convivir y de conmorir.

Estas inquietudes dieron origen, en una perspectiva necesariamente modesta, a los dos talleres propuestos a la Universidad Intercultural de Chiapas en la primavera del año 2006 y en 2011, también durante la primavera. Buscaron dichos talleres poner en práctica la idea de la interculturalidad como diálogo horizontal y el reconocimiento de la alteridad como valor: si es cierto que la diferencia puede ser un abismo, había que poner a prueba el abismo.

Partieron también de un supuesto indispensable a su puesta en marcha: la existencia de la nación como una comunidad política que incorpora comunidades culturales. Un presupuesto que no excluye, sino antes bien plantea, la necesidad de discutir en qué calidad las incorpora: si las avasalla bajo las diversas modalidades de la asimilación, intenta eliminarlas y genera entonces resistencias, o propicia espacios respetuosos de la diversidad.

No cabe duda de que este supuesto es discutible a profundidad y desde una multitud de enfoques. Uno de los que más impacto tiene es el que considera la nación como diluida en el mundo contemporáneo por el avasallamiento de las soberanías de los Estados a manos de instancias transnacionales postimperiales. Puede, desde esta óptica, parecer antitético considerar como comunidad política una entidad que - se dice- se diluye. Desde el punto de vista de una historiadora, cabe 
sin embargo formularse la hipótesis de que la nación diluida sigue siendo una comunidad política.

Impacto fuerte tiene también la perspectiva que considera el resurgimiento de los nacionalismos étnicos, que en las últimas décadas se han planteado como reacción a la mundialización y a la dilución, y aun desaparición, de los Estados-nación, en calidad de esferas voluntariamente impermeables y en buena medida excluyentes. Desde esta perspectiva, la cultura que define a una nación sería una y unificante. Parece, desde este punto de vista, un contrasentido definir la nación como comunidad de culturas. Aunque México no conozca reivindicaciones nacionalistas de corte étnico, como las que - fundamentalmente europeas - abonan esta visión, las elites que han dominado nuestro país sí han pretendido en diversos momentos de su historia contribuir a unificar la cultura mexicana, considerando las distintas culturas vivas en el territorio, así como otros tantos puntos de fuga de la unidad nacional.

Precisamente porque el supuesto de la existencia de la nación es discutible, cabe explicitar, en sentido histórico e historiográfico, el interés de considerarlo como punto de partida: la nación —esa construcción discursiva aunque también política- en el caso mexicano se trata de una comunidad política que tiene casi dos siglos en vías de construcción. A esa comunidad y a esa construcción se las pueden asociar muy disímbolos epítetos, como los asociados con la inequidad y la discriminación - los que subrayan su carácter inacabado, los relativos a la injusticia y a la dominación, a la exclusión o a la inclusión selectiva-; porque existe; se pueden subrayar sus límites en extensión y en profundidad, porque existe.

Desde este punto de vista, la historia de dicha comunidad de comunidades existe, aunque se represente de manera desigual en su escritura, o aunque poco de esa diversidad alcance un lugar en la historiografía y menos aún llegue a las aulas en las que la mexicanidad común, que tiende a ser monocroma, se construye. Por cierto, esa monocromía es sin lugar a dudas uno de los problemas mayores de la enseñanza de nuestra historia.

Por esa razón, uno de los ejes de renovación de la didáctica de la historia en nuestro país pudiera ser el proporcionar un mecanismo de apropiación de la historia nacional a través de ejercicios de escritura. Estos ejercicios requerirían de dos momentos iniciales de elaboración colectiva: un primer momento que conduzca a la comprensión de la historia como escritura a partir de una realidad, antes que como una realidad inmutable; y un segundo momento en el que se examinen el concepto de nación y el conjunto de representaciones que le están asociadas desde la perspectiva de los participantes. La consideración de ambos elementos abre la puerta a la explicitación de horizontes culturales e identidades múltiples, lo cual contribuye a evidenciar la complejidad histórica de eso que llamamos nación o de lo que calificamos como nacional.

Importa insistir en las dificultades de esta escritura y de la interculturalidad como planteamiento teórico, metodológico y político inacabado, con límites propios $\mathrm{y}$, sobre todo, con un horizonte utópico fuerte. ${ }^{10}$ Convendría sin duda - aunque no parece una actitud en vías de generalización- invertir la función de este horizonte y pensar la interculturalidad no como panacea, sino como punto de partida indispensable mas no suficiente. En calidad de punto de partida, la noción de interculturalidad plantea a la historiografía mexicanista algunos problemas que vale la pena abordar:

Un asunto de primer orden son los límites del lenguaje disponible. Aplicado al caso mexicano, el planteamiento de la interculturalidad - como por cierto el de la multiculturalidad - supone en general la coexistencia de dos matrices culturales confrontadas: una que podemos simplificar como amerindia y otra que podemos llamar, simplificando también, occidental.

Desde una perspectiva histórica, ambos términos designan realidades excesivamente amplias. Con 
facilidad vemos que, en particular el segundo, no encuentra obstáculos para deslizarse hacia lo "europeo" - aún excesivo en su amplitud - ni para concluir en lo "hispánico" — sobre cuya composición, por cierto, mucho hay por discutir, pero esto rara vez se hace de este lado del Atlántico, siendo como es el pan de cada día en la España contemporánea-.

El término "amerindio" en cambio — sin ignorar que tiene una historia propia - resulta en este marco tal vez sólo una forma un tanto menos impolítica que simplemente "indio" y, por lo demás, rara vez termina por deslizarse hasta muy específicas culturas cuyos nombres, sin embargo, no nos son desconocidos.

La reducción de identidades múltiples a la categoría "indio" tiene una historia de varios siglos; una historia compleja, pues no se trata de una reducción inmediata ni absoluta: los primeros cronistas europeos suelen aún consignar identidades específicas y hablan de nación tlaxcalteca, otomí, pime, tepaneca y un largo etcétera; una atención a la diversidad que sólo intelectuales de la talla de Francisco Xavier Clavigero ${ }^{\text {ll }}$ conservarán hacia el ocaso del dominio español (Clavijero, 1991).

La condensación de decenas de identidades específicas en la categoría "indio", que tan lúcidamente analizó Guillermo Bonfil como parte de la construcción colonial (Bonfil, 1972), es un proceso -igualar a los diferentes por la común condición de colonizadosque redondean sin escrúpulos los constructores de la nación independiente y que, como también lo sugiere Bonfil, puede leerse en términos de colonialismo, ese que forma parte de las relaciones sociales de dominación en el seno de cada país..$^{12} \mathrm{El}$ concepto "indio" es así un brocado de colonialismos cuya complejidad no radica sólo en el entretejido de unas y otras dominaciones, sino también en el hecho de que la categoría "indio" ha sido recuperada políticamente por los propios interesados con reivindicaciones específicas.

En lo puramente contemporáneo, el problema suele en parte presentarse en términos inversos: cuando los académicos se acercan a las comunidades indígenas, resulta difícil desprenderse del término "occidental" para designar a lo "no indio", pero en cambio este "occidental" tiene frente a sí comunidades culturales designadas con apelativos específicos. ${ }^{13}$

No cabe duda de que en la actualidad nos hace falta urgentemente una palabra. La causa de que nos haga falta es una paradoja histórica sólo aparente: la nación mexicana se construyó discursiva y materialmente apoyada en un mestizaje cuya definición se basa, por defecto, en la persistencia de los no mestizos, que son los indios. Excluidos en la práctica de la nación, los indios conservan esa identidad frente a una mexicanidad que los excluye. Como, sin embargo, en teoría la nación sí los incluye, no es posible designar la alteridad frente a lo indio con el término "mexicanos". Además, cada vez es más evidente la poca utilidad - $\mathrm{O}$, si se prefiere, la insuficiencia- del término "mestizos" para designar esa alteridad. Así pues, es patente la falta de un vocablo que permita designar a los mexicanos incluidos en el proyecto de nación - y aquí cabe recordar que ni la inclusión ni la exclusión obedecen a criterios puramente étnicos - frente a los grandes excluidos en la práctica, que la definición teórica constitucional sí incluye. La práctica académica ha recurrido al término "occidental", que no puede sino ser insatisfactorio - y cuyo uso además evidencia a qué punto el Occidente es una realidad cultural más que geográfica, semejante en nuestro continente a un gran gruyère -; pero aún podría con más imaginación recurrirse a los términos que las lenguas dominadas emplean para designarnos puesto que sí los tienen. Así, por ejemplo, las lenguas mayas emplean el término kaxlán; sólo un amor propio arrogante se incomodaría por ser tratado genéricamente de kaxlán por aquellos a quienes genéricamente llamamos indios.

Otra dificultad mayor que se plantea en la escritura de la historia si se busca incorporar las ambiciones de los proyectos llamados interculturales, es el estudio del conflicto. Parte importante del problema radica en la percepción de la interculturalidad como panacea 
capaz en sí misma de erradicar conflictos cuyas raíces son históricas - conflictos no sólo étnicos, sino también lingüísticos, religiosos y de género, entre otros-y que, precisamente, son conflictos interculturales. Resulta entonces indispensable invertir la perspectiva de la interculturalidad como panacea para plantearla como un simple punto de partida. Se impone así la admisión de la realidad intercultural como una realidad histórica conflictiva en distintos niveles que precisa ser analizada en su complejidad. Es conflictiva, de entrada, porque comporta y disimula relaciones de dominación que tienen siglos entretejiéndose y cuya sofisticación material y simbólica es creciente y continua.

El campo de la escritura de la historia abre un primer espacio para, más allá de la reivindicación de las ausencias, abordar sin ambages lo que constituye uno de los principales retos de la interculturalidad como planteamiento político y educativo: la necesidad de nombrar y analizar el conflicto —en su multiplicidad de rostros - para no ignorar su existencia y papel históricos o, peor aún, simular o suponer su inexistencia.

Pero nombrar el conflicto y analizarlo no bastan para pretender que se escribe historia interculturalmente - un objetivo probablemente rayano en lo utópico-; $y$, sin embargo, no es irrealista pensar en un ejercicio de síntesis que incorpore voces plurales y que al menos sea el producto de un diálogo abierto. La principal premisa de este diálogo y del ejercicio de escritura consecuente es que: intercultural es la realidad de dominación que ha prevalecido por siglos, mucho antes de ser un planteamiento académico.

\section{El mosaico de historias chiapanecas}

Con esta discusión en el horizonte se trabajó, de febrero a junio de 2011, en el taller "Mosaico de Historias Chiapanecas". ${ }^{14}$ Una de las ambiciones mayores de este taller era realizar un ejercicio de escritura donde ocuparan un lugar central las prioridades de investigación y de interpretación de una joven generación. Así fue como la agenda de trabajo y de escritura se estableció con arreglo a los intereses de un grupo de 16 jóvenes chiapanecos. ${ }^{15}$

En el privilegiar los intereses de una generación joven había más que una preocupación didáctica: de manera general, cuando se trata de recuperar saberes históricos no escritos o escasamente representados en lo escrito, se acude a la memoria de las generaciones mayores. Los adultos mayores - y señaladamente los varones - poseen el privilegio de la última palabra. El sentido de horizontalidad que preside la propuesta de una escritura intercultural de la historia exige que, sin que se pierda el respeto por la memoria de los mayores, se reconozca y respete la existencia de otras memorias posibles. ${ }^{16}$

Otra de las premisas del taller fue la de propiciar que los temas de las historias fueran determinados en absoluta libertad por sus autores; es decir, partimos de la convicción, con Marc Bloch, de que "todo es historia". Sin cortapisas, los participantes eligieron temas que fueron acotándose en el curso del taller. La gran mayoría de los temas se refiere a los lugares de origen o de residencia de sus autores, pero casi todos se alejan de lo que convencionalmente se esperaría de un texto histórico. Los autores están atravesados de una curiosidad que puede calificarse de etnográfica y lo que más les atañe es la forma en que la historia está presente en sujetos vivos. ¿Por qué se ha perdido lo que antes fue: vestidos, música, lengua, usanzas? ¿Cómo fue aquello de lo que sólo queda ahora el recuerdo en la memoria de los mayores: formas de hacer, de vestir, de trabajar, de relacionarse? ¿Cómo se entretejen lo material y lo simbólico en la vida cotidiana de pueblos concretos? ¿Qué usos dan las distintas generaciones a los mismos espacios y cómo caben las diferencias - y se abren sitio- en los contextos convencionales?

Una parte básica del trabajo de escritura desatado a partir de estas preguntas y otras cercanas fue realizada en el aula; importaba mostrar la trascendencia de desarrollar la capacidad analítica y narrativa de cada 
uno de los participantes, literalmente a papel y lápiz, y de manera independiente al uso de los infinitos recursos que los sistemas de información virtual ponen actualmente al alcance de quien sabe utilizarlos. ${ }^{17}$ La estrategia fue la redacción de un primer texto, de elaboración estrictamente personal, en el que se plasmó lo sabido sobre el tema elegido. Este texto permitió, especialmente, evidenciar lo por saber y mostrar los caminos posibles para indagarlo.

La segunda fase, central, consistió en visitar colectivamente los escenarios de las historias y en realizar recorridos de campo y también entrevistas colectivas con herederos directos de las historias. Cada visita fue preparada con anticipación por el principal interesado: el autor o autora de la historia. Siempre que fue posible, en el curso de las visitas tomamos espacio y tiempo para trabajar en taller en torno a la historia investigada. Ahí cobraron importancia las capacidades de observación de todos y cada uno y las interrogantes que cada quien formuló, que no siempre estaban presentes en el ánimo del autor responsable del tema.

Con San Cristóbal de Las Casas como centro de operaciones realizamos un conjunto de recorridos que son la urdimbre de este mosaico. En esas rutas captamos una muestra de la diversidad y riqueza del paisaje chiapaneco en sus profundos contrastes, andando siempre de familia en familia, pues las familias de los participantes fueron en cada sitio nuestras generosas anfitrionas. Así, los primeros recorridos nos condujeron a Amatenango del Valle y a Teopisca. En Amatenango, pueblo pendiente del ritmo del maíz - cuyos barrios se extienden a ambos lados de una carretera bordeada de artesanías y puestos de venta de elotes, y se disimulan a la vista en el paisaje - están los hornos en el seno de las viviendas, y las mujeres - pues ellas son las alfarerasmoldean pacientemente, sentadas en el suelo, figuras y tinajas.

En Teopisca, ese lugar de paso cuyo corazón cruza el viajero obligadamente, constatamos lo bien fundado de la fama de su gastronomía pródiga en sabores, el vínculo de ésta con el arte de recibir y lo escurridizo aún de un pasado en torno al cual también la fantasía se hace pródiga. Más adelante, en Comitán, advertimos el coqueteo constante con la frontera, que ya la presencia del retén militar anuncia, y otra vez topamos con una historia de contornos lábiles, donde figura tanto como se desdibuja una mujer: Josefina García, entre memoria y olvido, en la frontera del mito.

Mucho más seguro de sí encontramos el discurso sobre el pasado en Zinacantán, donde el diálogo fue y vino sin dificultad de los tiempos de la Conquista hasta el presente. Ahí fuimos invitados a advertir el relieve, no sólo de la historia zinacanteca, del entramado de relaciones entre religión, política, economía y vida cotidiana, sino también del interés que este pueblo ha suscitado, por décadas, en los estudios académicos, y del cual sus habitantes son más que conscientes. Bernardino López de la Cruz, nuestro compañero y guía, actúa con el convencimiento de que es tiempo de pasar a otra etapa: para él, la historia de Zinacantán debe ser escrita por los zinacantecos.

También en Simojovel la conciencia del entramado de relaciones económicas y políticas es aguda, en tiempos en que pesa el interés internacional creciente por la producción local de ámbar. Ésta se enmarca, para algunos todavía hoy, en un conjunto de representaciones sagradas relativas al uso de los recursos del medioambiente. Esta concepción del mundo entra en confrontación con la llegada impetuosa de cristianismos no católicos, como en otras regiones del estado de Chiapas.

En los espacios campesinos constatamos la vigencia de una división del uso de la palabra por género. Las mujeres campesinas comparten su palabra en ese espacio privilegiado que es la cocina. Fuera de ella, la palabra masculina domina y aún se impone como única, como sucedió en El Corralito, donde ellas acompañaban con movimientos del cuerpo el discurso del varón, asintiendo o discordando, entusiasmándose sin pronunciar palabra, incluso ante preguntas que 
directamente les fueron formuladas. En la cocina es distinto, en torno al metate y al fogón la palabra de las mujeres circula, va y viene, fuera de la rigidez de la entrevista, para adentrarse en aspectos fundamentales de la vida: las uniones y los sinsabores, el ir y venir de los hijos, los sabores y los saberes de la vida diaria. Es, por lo general, una palabra que se teje en torno a un gesto que generosamente comparte, ofrece un cuenco de pozol, pencas de guineos, o prepara comida para los recién llegados. Así fue en Amatenango, así fue en El Progreso, así en El Corralito, así también en Nuevo Mérida. La presencia entre nosotros de mujeres que sin timidez supieron entrar en las cocinas y sumarse a estas labores fue fundamental para tender el puente de comunicación en cada caso, y no sólo, por cierto, en los medios campesinos.

Asimismo, la presencia de hablantes de tseltal, tsotsil y chol entre los integrantes del taller fue indispensable para rebasar los límites del español como lengua franca y permitió una cercanía con nuestros anfitriones que de otra manera hubiera sido prácticamente imposible. En algunos casos, como en El Corralito y en Nuevo Mérida, la labor de las intérpretes fue imprescindible: así, las entrevistas, realizadas en tseltal en el primer caso y en chol en el segundo, fueron traducidas simultáneamente al español.

En otros sitios, en cambio, constatamos cómo el español en su dominio lo ha avasallado todo y aun borrado. Así, en El Porvenir, de la lengua mam queda la nostalgia y un grupo tenaz, aunque pequeño, de adultos mayores que intenta dar nuevo impulso al habla de su lengua entre las nuevas generaciones. Se vincula la defensa de la lengua a la vida y práctica de la música - aunque no se canta en mam-, especialmente de la marimba, amenazada en las últimas décadas por los principios propagados por Iglesias de implantación reciente en la zona que consideran negativa la práctica de la música fuera de lo ritual, y en consecuencia la del baile.

Bajando a Bella Vista, no falta quien haga referencia a la lengua mam sin que nadie la hable. En cambio, la marimba ocupa un lugar preponderante. Nuestros anfitriones esperan la menor provocación para ejecutar piezas a dos, cuatro, seis manos, involucrando a varias generaciones, hombres, niñas y niños. A pocos kilómetros de ahí, en el ejido El Progreso, donde nos hospedamos, nuestro anfitrión, dedicado por entero al cultivo del café como la mayoría de los pobladores de la región, sabe tocar marimba, pero ha dejado de hacerlo por motivos religiosos. Uno de sus hijos, en cambio, compone música religiosa que interpreta en órgano electrónico y reproduce en discos para su comunidad. Un temperamento profundamente musical prevalece en la región. Como constatamos en El Porvenir, no es que algunos habitantes sean músicos de profesión, es que la música es parte de la vida de las familias a través de cada uno de sus miembros.

En varios de los lugares que visitamos pudimos constatar aquello que saben bien y comentan los chiapanecos de diversas procedencias: las personas mayores conservan la lengua de sus antepasados, que la generación de sus padres o la de ellos mismos fue dejando de hablar por efecto de políticas educativas "mexicanizadoras" y por la violencia castellanizadora de las escuelas mal llamadas bilingües que reprimieron el uso de la lengua materna - políticas que apenas en nuestros días parecen iniciar un giro en otro sentido, y que cuentan entre sus resultados con la ruptura del relevo generacional de transmisión de las lenguas maternas, sin que sea seguro que con la presencia de los mayores esta falta de eslabones pueda compensarseAsí fue como en Copainalá nos hablaron de que la lengua zoque se hablaba... lo que hoy queda es la conciencia aguda de la pérdida.

A estas lenguas que se hablaron y que viven en peligro se suma en la región del Soconusco, en Acacoyagua, la lengua japonesa, que todavía hoy al menos doña Yoshiko habla y lee, si bien no parece que otras personas del pueblo puedan hacerlo. La entrevistamos en el oasis de su corredor rodeado de jardín, generosa en su saber, orgullosa de su pasado 
japonés, agradecida con la tierra que recibió a estos migrantes del lejano Oriente hace ya más de un siglo, $\mathrm{y}$ con sus habitantes.

Era del interés de nuestro taller explorar lo sucedido con los trajes tradicionales chiapanecos en algunas comunidades específicas. En Nuevo Mérida fue el objetivo mayor de nuestra visita; en Amatenango, uno de los principales. Es evidente que en todo Chiapas el vestido elaborado a mano ha cedido terreno a la ropa de factura industrial. Sin embargo, la potencia de la representación por el vestido sigue siendo grande, especialmente para las mujeres. En Zinacantán es mayúscula. En los lugares donde el vestido tiene o ha tenido importancia nos hablaron del tema, con autoridad, los hombres; allí donde era nuestro interés principal requerimos el punto de vista, indispensable, de las mujeres y encontramos una valoración vinculada no sólo a lo estético y a lo simbólico, sino a aspectos de la vida práctica cotidiana, como la disponibilidad o carencia de materiales y modelos.

En Acacoyagua, en El Porvenir, en Nuevo Mérida, encontramos personas conocedoras en profundidad de un pasado de migraciones para fundar nuevos asentamientos o nutrir uno ya existente; en El Progreso, la valoración de una mano de obra transfronteriza y temporal, que hace posible el cultivo del café. En las distintas localidades visitadas constatamos el vínculo amoroso con la tierra y, como tal, también conflictivo.

En todos los sitios visitados son visibles los efectos de la implantación en décadas recientes de Iglesias que compiten con la presencia secular del culto católico, algo que se traduce en confrontaciones en torno a las prácticas tradicionales y en la invención de nuevas tradiciones.

En ciertas comunidades pequeñas, comprobamos la tensión en torno a la presencia de forasteros indagadores, mitigada en nuestro caso por el hecho de ser invitados directos de alguna familia. Fueron notorias las tensiones en Nuevo Mérida, y su magnitud nos desaconsejó visitar Pozuelos, en el municipio de Chamula.
Asistimos así, en el sentido intenso de la palabra, a una nueva forma de confrontación: la formación universitaria de los jóvenes y en especial de las mujeres, motivo de orgullo para sus familias, que así lo manifestaron al recibirnos con toda generosidad, pero también de preocupaciones y aun de conflicto con otras familias. Estas tensiones viven abiertas en el presente como una gran incógnita, generadora tanto de esperanza como de conflicto.

Abordamos la ciudad de San Cristóbal desde miradores diversos: el pasado cercano en la memoria de generaciones vivas, la trayectoria de una de las primeras estaciones radiofónicas, la diversidad sexual y las tácticas de que dispone para reclamarse parte del paisaje de una ciudad de afamado - pero resquebrajadoconservadurismo, de trazo antiguo resignificado por el turismo internacional. Una ciudad que los jóvenes hacen suya de manera impetuosa desde que en 1994 irrumpiera en sus calles el EZLN, convirtiéndose en referencia histórica para varias generaciones según puede verse en los textos centrados en aquélla que las nuevas generaciones llaman "Sancris".

En una tercera fase, retornamos al aula para revisar los avances y continuar escribiendo. Huelga decir que los autores siguieron realizando entrevistas e indagaciones por cuenta propia. En la medida de lo posible, se hicieron lecturas cruzadas de los diversos textos, si bien nunca se alcanzó el objetivo de que todos los participantes leyeran todos los textos.

El producto del taller, un conjunto de 16 textos de variadas temáticas y pronto a publicarse, evidencia la vecindad y la complicidad indispensable de la historia con otras disciplinas, muy señaladamente con la antropología. Pone de manifiesto, en la línea de lo abonado desde hace varias décadas por la llamada historia oral, que la renovación de una disciplina tan dominada por las jerarquías de lo escrito pasa por correr riesgos diversos. En este caso, el rasgo y el riesgo principal es el que representa una escritura sin pretensión de asepsia, cuyo punto de partida es 
el diálogo con sujetos vivos. Un diálogo del cual no necesariamente sale fortalecida una "verdad" en el sentido positivista del término, sino una relación viva, y por tanto verdadera, de sujetos concretos con su pasado reconstruido y recreado, un pasado hecho propio.

El resultado, no por modesto, es menos provocador: muestra, en primer lugar, la variedad de temas que para una generación resultan en un momento preciso relevantes y dignos de ser objeto de indagación y de escritura. Así, sobre San Cristóbal de Las Casas, el interés se centró en las transformaciones de la vida y de los usos de la ciudad, en particular después de 1994; en las formas actuales de apropiación creativa del espacio urbano por parte de las y los jóvenes; en los espacios que logra la comunidad gay en esta misma ciudad; así como también en la historia de la radiodifusora XERA, una estación pionera.

Fuera de la llamada capital cultural de Chiapas, la atención del taller se enfocó hacia los quehaceres cotidianos en Amatenango del Valle; la pérdida de la vestimenta ch'ol en la comunidad de Nuevo Mérida; la inmigración japonesa y su huella en Acacoyagua; la memoria escurridiza de Josefina García, heroína de Comitán; la historia general de Teopisca; la importancia del café y de su feria en Bella Vista; la música mam y la identidad cultural en El Porvenir; la producción de ámbar y el mundo no sólo económico, sino también simbólico, que la rodea en Simojovel; la tradición de los linajes indígenas en El Corralito; las transformaciones de la comunidad de Pozuelos en las décadas recientes; la vida contemporánea de los zinacantecos y, en todos los casos, las formas de relación viva con el pasado de distintas generaciones.

Como se puede apreciar, los temas van desde la médula de la vida campesina, hasta aquello que por largo tiempo ha sido considerado simplemente folclórico - las formas de vestir o las fiestas de los pueblos-o aun accesorio - la vida afectiva y las orientaciones del placer- ¿. ¿Dónde está el corazón? tendría que ser la pregunta consecuente.

\section{Si algo se puede concluir...}

Hace tiempo que la nación ha dejado de ser vista como un marco natural de la escritura de la historia, en la medida en que se ha puesto énfasis en su propia historicidad, subrayándose la parte que tienen actores concretos en su construcción objetiva y subjetiva; pero también, y sobre todo, en la medida en que han probado fecundidad, en el trabajo de investigación histórica, otros marcos espaciales y temporales, así como enfoques que privilegian a actores cuya razón de ser última no es, ni con mucho, la nación. Estas formas de hacer historia diversifican a la nación, la rebasan o aun la ignoran. Y, sin embargo, esta realidad política contemporánea sigue presente como un tópico de reflexión necesario para quien se interesa por los procesos históricos.

La práctica de la historia, por su parte, enfrenta cotidianamente el conjunto de retos planteados a las formas de escritura del devenir humano en sociedad, en que se juega su capacidad de renovación como disciplina. Retos que tienen que ver con el lugar del pasado en el presente de las generaciones vivas, con la capacidad de los historiadores para dialogar con la memoria de dichas generaciones, con la relación estrecha de la disciplina con la escritura, con su capacidad de producir saberes.

A las múltiples confrontaciones de que ha sido objeto la práctica de la escritura de la historia nacional es preciso sumar, de manera urgente, la que comporta la aceptación del carácter intercultural del devenir histórico de las naciones y, específicamente, de la nación mexicana.

Los resultados del taller "Mosaico de Historias Chiapanecas" sugieren que, ante el rotundo fracaso —ese sí, nacional - de la didáctica de la historia, ${ }^{18}$ la apuesta por una apropiación basada en un ejercicio comprometido de escritura - en el sentido del empeño personal y de la asunción del riesgo que representapuede ser fructífera. En este caso, los textos así elaborados, sin tenerlo por objeto central, evidencian la huella de la historia nacional en el transcurrir de la 
vida cotidiana como un flujo multifacético de violencia recurrente.

Los frutos de este taller se suman a los esfuerzos de aquellos colegas que, por diversas vías, contribuyen a modificar las formas en que se escriben nuestras historias. En ese mismo sentido, una historia nacional en curso de renovación corre el conjunto de riesgos que supone el incorporar las aportaciones de diversas corrientes, quizás inauguradas en el gesto - antropológico, por cierto- de Eric Wolf (Wolf, 1987) y sus reflexiones sobre la gente "sin historia", y en México precedidas desde el porfiriato por la afirmación de Agustín Rivera sobre la capacidad política de indios y campesinos (Rivera, 1954: 151-154). No puede esta renovación dejar de considerar los debates abiertos en el campo científico y en el político — aquí definitivamente indisociablespor los estudios llamados subalternos y postcoloniales, así como por el feminismo, en su exigencia planteada a la escritura de la historia en términos del reconocimiento de los múltiples sujetos que intervienen en ella.

¿Cómo se leerá lo nacional desde esta escritura? Seguramente de maneras complejas, interculturales y por tanto conflictivas. Nada más indeseable aquí que una receta.

Un elemento básico me parece desprenderse con claridad de la experiencia modesta del taller "Mosaico de Historias Chiapanecas": una nueva escritura de la historia debe prescindir de jerarquías, no sólo en términos de la definición de los temas por abordarse, sino, y sobre todo, de los sujetos y de la calidad de su palabra. La agenda de la historia nacional pendiente de indagarse y escribirse bien puede empezar a establecerse a partir de las inquietudes de quienes, sin acompañarse de la etiqueta de expertos, señalan agudamente páginas en blanco.

\section{Notas}

${ }^{1}$ Las marcas cronológicas de principio y fin de siglo que Hobsbawm propone corresponden a dos momentos de desencantamiento del orden internacional no sólo occidental: la cruenta guerra iniciada en 1914, que segó el optimismo europeo y su creencia en un progreso, y lo que él llama el "fin de la era soviética", en 1991, que acabó no sólo con el llamado "socialismo real", sino con un paradigma alternativo al capitalismo. No recurre Hobsbawm al término de "desencantamiento", pero sí subraya el decaimiento del ánimo general, en particular con la guerra de 1914-18.

2 En sentido político y en sentido histórico, el endiosamiento de la nación conoció su cénit en el siglo XX con el apogeo y extrema beligerancia de los nacionalismos, tema sobre el cual han corrido ríos de tinta. En sentido historiográfico, sin embargo, la nación como figura y como creación tiene mayor potencia durante el siglo XIX, cuando la historiografía cumple con la misión de dotar a la nación como creación política de un pasado nacional. A este siglo corresponden las grandes empresas occidentales de creación de historias nacionales. Los nombres europeos de Leopold von Ranke o Jules Michelet - por citar los más emblemáticos aunque distintos entre sí- tienen de este lado del Atlántico sus correspondientes en el chileno Diego Barros Arana o en el mexicano Vicente Riva Palacio. Sobre el tema puede verse Palti, 2003.

3 Cuando analiza las formas de la escritura nacional de Riva Palacio y de su equipo en México a través de los siglos, Ortiz Monasterio (2004) destaca la importancia de la cercanía de la guerra y la fragilidad de la nación para todos cuantos participaron en la empresa de escritura: la patria sobre la que escriben es aquella por la que en su momento algunos se juegan la propia vida. Nada más alejado del ideal y supuestamente idóneo distanciamiento del historiador con respecto a su objeto de estudio; asimismo, nada más alejado de la relación afectiva de los intelectuales contemporáneos con el concepto de patria, por el cual puede hacerse la hipótesis de que muy pocos estarían dispuestos a arriesgar la existencia. 
${ }^{4}$ Este debate ha tenido por escenario principal los espacios académicos de Europa —señaladamente Francia-y los Estados Unidos, aunque ha repercutido en los espacios académicos latinoamericanos que siguen siendo ampliamente subsidiarios en la materia. No se pueden ignorar las radicales interpelaciones del linguistic turn, cuyo exponente más conocido y también más caricaturizado es Hayden White (1992) —aunque dista de ser el único-, ni las mediaciones y matices aportados por los intelectuales franceses, más próximos en su conjunto a la defensa del estatuto de la historia como productora de conocimiento sobre la realidad. Cabe señalar el esfuerzo de diálogo de autores como Roger Chartier (1998) y el monumental trabajo de síntesis y propuesta filosófica de Paul Ricoeur (2000). También Carlo Ginzburg, desde una perspectiva "a ras del suelo" como él mismo gusta de calificarl,a y retomando las reacciones pioneras de Arnaldo Momigliano, ha enriquecido el debate (Ginzburg, 2000 y 2006). En México, el espacio de la revista Historia y Grafía es quizás el único que ha seguido de manera sistemática este debate.

${ }^{5}$ De manera dramática y penetrante, una preocupación conexa por los límites de la representación historiográfica se había expresado desde el coloquio efectuado en Los Ángeles en 1990 y cuyo debate puede verse en Friedlander (1992).

${ }^{6}$ Cierto que, desde la perspectiva aquí elegida, no deja de plantearse la pregunta sobre el sentido que tiene que una sociedad se apropie la elaboración de un discurso escrito - la historia - sobre una realidad - la naciónque a su vez es un discurso.

7 En el marco de un texto como el presente, es prácticamente imposible proporcionar un balance historiográfico de una materia tan basta en la medida en que una parte considerable de la producción historiográfica mexicanista, como la de otros países, tiene a la nación por marco. A veces, incluso aquella que en sentido estricto debería poder escapar a él por referirse a periodos anteriores a la creación de una nación mexicana. El marco nacional se impone y se constituye en una prisión historiográfica no sólo porque corresponde al marco histórico-cultural de más de siete generaciones de historiadores y se erige en un molde del imaginario, sino porque también técnicamente el historiador es presa de la organización de los acervos documentales - archivos y bibliotecas - que hacen su investigación posible.

${ }^{8}$ Soy de la opinión de que el proyecto mexicano de educación intercultural obedeció a decisiones políticas coyunturales más que a una reflexión, programación y definición de fondo. A pesar de eso, ha movilizado a sujetos de gran capacidad y talento y, en la práctica, abrió espacios educativos inéditos cuyo impacto en sociedades concretas es necesariamente variable y está aún por evaluarse. En esos espacios, precisamente, más allá de las vicisitudes de la política del día, porque constituyen recursos para escapar a ella y abrir grietas en el sentido en que las piensa John Holloway (2011), los valores planteados por la educación intercultural resultan esperanzadores.

${ }^{9}$ Un esfuerzo notable que explora estas relaciones entre diferentes precisamente en territorio chiapaneco $-\mathrm{y}$ antes de que lo intercultural se pusiera de moda- es el de Viqueira (2002).

${ }^{10}$ Sarah Corona Berkin opta por separarse del concepto de "interculturalidad" - prefiriendo el término "entrecultura" - por su connotación de "generalidad, equidad y reciprocidad" que elude el conflicto, y explica: "De la reflexión intercultural me inquieta que el centro de la discusión sean las culturas y no el diálogo sobre las diferencias en el espacio público" (Corona, 2007: 13).

${ }^{11}$ Presentando la biografía del historiador que hiciera Juan Luis Maneiro, SJ, el también historiador jesuita Jesús Gómez Fregoso aclara: "Antes de incursionar en la biografía del historiador veracruzano, no está por demás comentar la ortografía del apellido Clavigero. Por lo común se escribe con j. Los catálogos jesuitas de los tiempos virreinales usan de manera indistinta Clavijero y Clavigero, pero consta, por manuscritos que 
he consultado, que el biografiado siempre escribía su apellido con g: Clavigero." (Gómez, 2004: 17).

12 La reflexión más potente sobre el colonialismo adjetivado como "interno" es la de González Casanova, quien reformuló el concepto (González, 2006).

${ }^{13}$ Así ocurrió en el trabajo que hizo posible el libro Entrevoces... elaborado entre maestros wixarikas y occidentales (Corona, 2007).

${ }^{14}$ El taller se realizó en el marco de mi estancia sabática en la Universidad Intercultural de Chiapas, con el apoyo irrestricto de quien fuera entonces su rector, el Dr. Andrés Fábregas Puig, así como del Mtro. Jaime Torres Burguete, director en ese tiempo de la División de Procesos Sociales. Para su realización resultó fundamental la experiencia del taller efectuado en 2006 con profesores.

${ }^{15}$ Participaron en el taller estudiantes de la licenciatura en comunicación intercultural, de la licenciatura en lengua y cultura, así como las profesoras Elisena Sánchez Román y Yoimy Castañeda Seijas.

${ }^{16}$ Desde una perspectiva de reflexión sobre las formas y las escrituras posibles de la historia de la nación mexicana, ya el taller llevado a cabo con niñas y niños de la comunidad indígena de Mezcala de la Asunción, Jalisco, en el año 2009, había mostrado la potencia de la memoria de las más jóvenes generaciones y el potencial de métodos de trabajo basados en el respeto de las formas de expresión y prioridades de aquellos que no ostentan en una comunidad o grupo una posición jerárquica. El resultado puede apreciarse en el libro Mezcala ise querían llevar la isla! (VV.AA., 2010), de la autoría de 106 niñas y niños de la citada comunidad.

${ }^{17}$ Estos recursos, en su inmensidad, son a la vez un campo abierto y una prisión para las facultades intelectuales en la medida en que, a la vez que multiplican exponencialmente las posibilidades de búsqueda, crean la convicción de que todo se encuentra en ellos y encerrado en ellos; así los sujetos, en una suerte de reflejo condicionado, antes que buscar en el propio cerebro, y aún despreciando el saber que en él pueda existir y generarse, acuden a la red.
${ }^{18}$ Para generaciones enteras de mexicanos, dos materias resultan manifiestamente repelentes: las matemáticas y la historia; la historia además se ve afectada por la etiqueta de inutilidad y el sentimiento de tedio. Esto es muestra del fracaso de las didácticas que la acompañan, no sólo a nivel básico, sino en la educación media superior.

\section{Bibliografía}

Bonfil Batalla, Guillermo (1972), "El concepto de indio en América. Una categoría de la situación colonial”, en Anales de Antropología, vol. 9, pp. 105-124.

Bourdieu, Pierre (2001), Science de la science et réflexivité, París: Raisons d'Agir Editions.

Bourdieu, Pierre (2002), Lección sobre la lección, Barcelona: Anagrama.

Certeau, Michel de (1978), L'écriture de l'histoire, París, Gallimard.

Chartier, Roger (1998), Au bord de la falaise, l'histoire entre certitude et inquiétude, París: Albin Michel.

Clavijero, Francisco Javier (1991), Historia antigua de México, México: Porrúa.

Corona Berkin, Sarah et al. (2007), Entrevoces... Fragmentos de educación "intercultural", Guadalajara: Universidad de Guadalajara.

Fernández Sebastián, Javier (dir.) (2009), Diccionario político y social del mundo iberoamericano, Madrid: Fundación Carolina/Sociedad Estatal de Conmemoraciones Culturales/Centro de Estudios Políticos y Constitucionales.

Friedlander, Saul (1992), Probing the Limits of Representation. Nazism and the "Final Solution", Cambridge-Londres: Harvard University Press.

Ginzburg, Carlo (2000), Rapporti di forza. Storia, retorica, prova, Milán: Feltrinelli.

Ginzburg, Carlo (2006), Il filo e le tracce. Vero, falso, finto, Milán: Feltrinelli.

Gómez Fregoso, J. Jesús, SJ (2004), "Introducción", en Maneiro, Juan Luis, SJ, Francisco Xavier Clavigero, 
SJ. Ilustre universitario constructor de la patria mexicana, México: Universidad Iberoamericana/ITESO, pp. 15-19.

González Casanova, Pablo (2006), "El colonialismo interno. [Una redefinición]", en Borón, Atilio A., Javier Amadeo y Sabrina González (comps.), La teoría marxista hoy: problemas y perspectivas, Buenos Aires: CLACSO, pp. 409-434.

Hartog, François (2005), Évidence de l'histoire. Ce que voient les historiens, París: Éditions de l'École des Hautes Études en Sciences Sociales.

Hobsbawm, Eric (1994), The Age of Extremes. The short twentieth century 1914-1991, Londres: Michael Joseph.

Holloway, John (2011), Agrietar el capitalismo. El hacer contra el trabajo, México: Sísifo/Bajo Tierra Ediciones/ Instituto de Ciencias Sociales y Humanidades Vélez Pliego de la BUAP.

Koselleck, Reinhart (2003), Aceleración, prognosis y secularización, Madrid: Pre-textos.

Koselleck, Reinhart (2004), historia/Historia, Madrid: Trotta.

Ortiz Monasterio, José (2004), México eternamente. Vicente Riva Palacio ante la escritura de la historia, México: Instituto José María Luis Mora/Fondo de Cultura Económica.
Palti, Elías (2003), La nación como problema. Los historiadores y la "cuestión nacional", Buenos Aires: Fondo de Cultura Económica.

Ricoeur, Paul (2000), La mémoire, l'histoire, l'oubli, París: Editions du Seuil.

Rivera, Agustín (1954), Anales de la vida del Padre Hidalgo, Guadalajara: Universidad de Guadalajara.

Sarlo, Beatriz (2005), Tiempo pasado. Cultura de la memoria y giro subjetivo. Una discusión, Buenos Aires: Siglo XXI.

Tischler Visquerra, Sergio (2005), Memoria, tiempo y sujeto, Guatemala: Instituto de Ciencias Sociales y Humanidades de la Benemérita Universidad Autónoma de Puebla/F\&EG.

VV.AA. (2010), Mezcala isequerían llevar laisla!, Guadalajara: H. Congreso del Estado de Jalisco.

Viqueira Albán, Juan Pedro (2002), Encrucijadas chiapanecas. Economía, religión e identidades, México: Tusquets/El Colegio de México.

White, Hayden (1992), Metahistoria. La imaginación histórica en la Europa del siglo XIX, México: Fondo de Cultura Económica.

Wolf, Eric R. (1987), Europa y la gente sin historia, México: Fondo de Cultura Económica. 